\title{
Management of Large Radicular Cyst by Surgical Endodontic Therapy: A Case Report
}

\author{
Carla Mendonça Augusto, ${ }^{1}$ Carolina Oliveira de Lima, ${ }^{1}$ Fabiola Ormiga Barbosa Soares, ${ }^{2}$ Heloisa Dell Santo Gusman ${ }^{2}$ \\ ${ }^{1}$ Department of Integrated Clinical Procedures (Endodontics), School of Dentistry, State University of Rio de Janeiro (UERJ), Rio de Janeiro, RJ, Brazil \\ 2Department of Dental Clinic, School of Dentistry, Federal University of Rio de Janeiro, Rio de Janeiro, RJ, Brazil \\ - Conflicts of interest: none declared.
}

\section{Abstract}

Objective: To demonstrate a surgical treatment of a large infected root cyst associated with the upper lateral incisor through a case report. Case Report: Patient reported a major complaint of swelling in the palatal region that was present for 6 months. The patient was submitted to non-surgical endodontic treatment. After clinical and radiographic examinations, decompression marsupialization was performed to create a window on the buccal cortical wall and an acrylic resin drain was installed in the depth of the cystic cavity. Two months later, a procedure was planned with cystectomy and apicoectomy on tooth 12, enucleated cyst, bone graft and barrier with purified collagen sponge. After enucleation, a sample of the cystic capsule was examined histologically and the diagnosis of periapical cyst was confirmed. Conclusion: After 1 -year, clinical cicatrization and radiographic resolution of the maxillary radiolucency were verified and the patient did not present painful symptoms, demonstrating the success of the treatment performed.

Keywords: Apicoectomy; Case report; Enucleation; Periapical diseases; Radicular cyst.

\section{Introduction}

$\mathrm{T}$ The periapical cysts are the most common odontogenic cystic lesions of inflammatory origin and the root cysts originate from epithelial residues present in the space of the periodontal ligament and their proliferation is activated by an inflammatory-type mechanism. ${ }^{1}$ They are generally symptomless but can result in a slow-growth tumefaction in the affected region ${ }^{2}$ and are normally diagnosed during a routine radiologic investigation. Radiographically, the classic description of the lesion is a round or oval, well-circumscribed radiolucent image involving the apex of the infected tooth.

The clinician must follow an organized thought process to arrive at an accurate diagnosis of an apical radiolucent lesion associated with the tooth in cases where the diagnosis is not clear, diagnosis is not clear. Moreover, the clinician could choose three-dimensional tecnhiques to better evaluate the lesion, such as cone-beam computed tomography (CBCT). ${ }^{3}$ Biopsy and histopathological analysis of the lesion can provide definitive confirmation of the diagnosis of a suspected lesion. ${ }^{4}$ However, a biopsy is recommended only if there are concerns about the clinical diagnosis, not to do as a routine. ${ }^{5}$

It is not yet well-established in the literature whether its treatment should be surgical or non-surgical. The treatment of periapical cysts includes conventional nonsurgical root canal therapy when lesion is localized or surgical treatment like enucleation, marsupialization or decompression when lesion is large. ${ }^{6}$ According to different techniques described in the literature, the surgical technique was selected as the treatment of choice for the patient, after the attempt of treatment with the non-surgical endodontic technique. So, this case report showed the successful surgical management of a large infected radicular cyst which was associated with maxillary lateral incisor.

\section{Case Report}

A 52-year-old female patient reported to the Department of Dental Clinic of the Federal University of Rio de Janeiro (UFRJ) with chief complaint of swelling in the palatal region, which had been present for 6 months. The dental history revealed repeated prescription of antibiotics and analgesics at private dental clinics in the previous months, indicated for the same persistent swelling. Medical history was unremarkable to the present case.

Intraoral clinical examination revealed a round to oval swelling soft and fluctuant, which was located on palatal region extending from teeth 11 to 15 . Spontaneous pus discharge from the fistula occurred in the vestibular region of teeth 12 and 13 . Thermal pulp vitality testing showed positive responses in 11 and 13. All teeth were non-tender to percussion test. Radiographic examination revealed a well-circumscribed, large unilocular and radiolucent area associated with teeth 11 to 13 . The endodontic treatment of tooth 12 was previously made by another dentist and presented good quality on radiographic examination.

Patient was subject to maxillary occlusal radiograph, cone beam computed tomography (CBCT) (Figure 1) and routine laboratory investigations that were within normal limits. CBCT revealed a lesion about $3 \mathrm{~cm}$ wide affecting the right premaxilla and contacting the floor of the nose and the maxillary sinus. A buccal cortical break and bone expansion 
were present. From the history, clinical examination and investigation, a provisional diagnosis of infected radicular cyst was made.

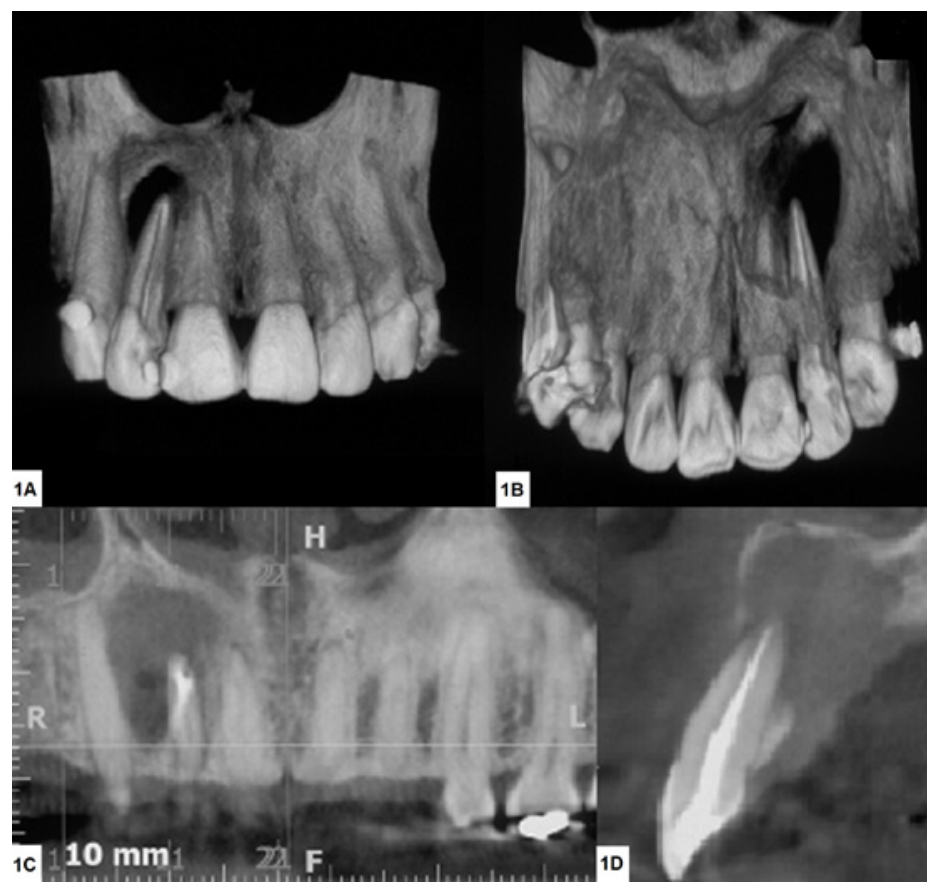

Figure 1. (A): 3D reconstructed pre-operative cone beam computed tomography buccal view and (B): palatal view (C): coronal and (D): sagittal plane.

A treatment plan was formulated and explained to the patient, after this, informed consent was formally taken. Marsupialization with decompression was planned by creating a window in the buccal cortical plate and a drain was positioned under local anesthesia.

After infiltration of anesthetic, a flap was created between the root eminences of teeth 11 and 13. During the marsupialization a hole of approximately $2.5 \mathrm{~cm}$ was created and an acrylic resin drain was inserted and installed at the depth of the cystic cavity, fitting perfectly to the surgical site. Two 3-0 sutures were placed through and surrounding to stabilize the drain during the initial healing. The patient was instructed to irrigate through the lumen of the drain 3 times a day with chlorhexidine $0.12 \%$, consistent with a published protocol.?

Two months later, the drain was removed and the mucosal orifice was sutured and radiographic examination revealed substantial but incomplete surgical endodontic healing therapy. Cystectomy and apicectomy of the tooth 12 were performed one month after the drain removal. The surgery was performed under local anesthesia, the vestibular flaps were removed, the enucleated cyst, bone graft (Alobone poros, Osseocon, Rio de Janeiro, Brazil) and barrier with purified collagen sponge (Surgidry dental, Technodry, Minas Gerais, Brazil) were performed (Figure 2).

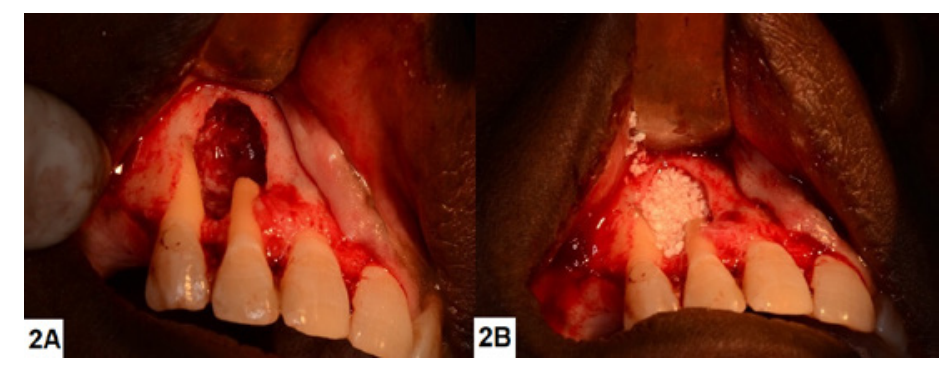

Figure 2. (A): Surgical picture after surgical enucleation of the cyst and apicoectomy of tooth 12. (B): Surgical picture after bone graft.

After enucleation, a sample of the cystic capsule was taken for biopsy and a complete histological study was made to exclude any other types of tumor. The histopathological report confirmed the previous diagnosis of an infected root cyst. Histological sections showed a capsule of dense connective tissue, predominantly chronic inflammatory infiltrate and partially covered by stratified squamous epithelium of odontogenic origin (Figure 3).

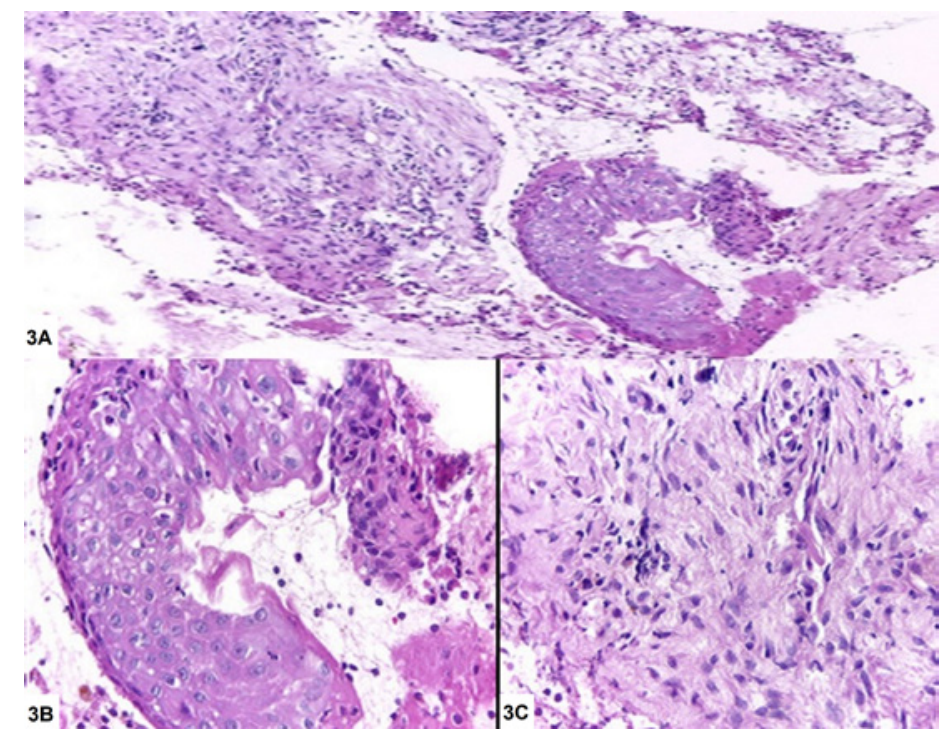

Figure 3. (A): Small fragment of stratified squamous epithelium and fragment of cystic capsule exhibiting moderate chronic inflammatory infiltrate. (B): Fragment of stratified squamous epithelium. (C): Fragment of cystic capsule exhibiting moderate chronic inflammatory infiltrate. (Magnifications: x100 and x400).

Clinical examinations at 1,3 and 6 months showed no sensitivity to percussion or palpation, and the soft tissues were healthy. After 12 months, clinical healing and radiographic resolution of the maxillary radiolucency were successfully completed (Figure 4).

\section{Discussion}

Intra-radicular or extraradicular infections can originate and perpetuate periapical pathological changes similar to inflammatory cyst. ${ }^{8}$ However, in the absence of aggressive agents, the immune system is able to initiate and develop 
repair of tissues and structures that have been affected in the pathological process, such as repair of a cystic lesion. ${ }^{9}$

Some previous reports have been successfully only with non-surgical endodontic treatment of cystic lesion. ${ }^{2,10}$ However, in the present case, only endodontic therapy was not sufficient for tissue repair, and therefore, surgical procedure was necessary to be made.

The treatment of choice depends on the size and location of the lesion, the bone integrity of the cystic wall and its proximity to vital structures. ${ }^{11}$ The marsupialization and decompression are techniques widely used for the primary or definitive treatment of cystic disease, ${ }^{12-14}$ because these techniques prevent damage to noble anatomical structures and reduce surgical trauma. ${ }^{13}$ In the present study, the techniques mentioned above were chosen, because the cystic lesion was close to the incisive foramen.

Different devices for decompression of cystic lesions and maintenance of the surgical window have been described in the literature..$^{15-17}$ After marsupialization, an acrylic resin drain was made and installed through an opening in the cortical plate of the buccal wall, which fitted perfectly to the surgical site. According to other studies, as shown in the present case, the decompression device was attached to the surrounding soft tissue with sutures. ${ }^{16,18}$

Decompression and marsupialization are procedures that have disadvantages such as communication between the cyst and the oral cavity, which can facilitate infection and require patient commitment. The patient should irrigate the cystic cavity through the drain and do frequent follow-up visits with the dentist ${ }^{19}$, as was done.

In the present case, the lesion was enucleated together with curettage, followed by apicoectomy of element 12 and allogenic bone graft to fill the defect caused by cystic lesion. The success in bone healing is directly related to the size of the bone defect, the anatomical location, the age of the patient and other parameters. ${ }^{20}$

A previous study ${ }^{21}$ evaluated large cysts and reported that there was no relapse after decompression treatment with subsequent cystectomy. Similar to results of the present study, that no recurrence of cystic lesion after treatment with decompression and cystectomy during the following year of the procedures was detected. In addition, healing of the periapical area was completed, showing the high predictability of this type of treatment.

\section{Conclusion}

The present case reported can confirm that marsupialization and enucleation along with bone graft had presented regenerative benefits along with good functional recovery after 1 year.

\section{Acknowledgements}

We would like to thank the Department of Pathology and Oral Diagnosis of Federal University of Rio de Janeiro for the support.

\section{References}

1. Ciulli E, Rocci M, Bollero R, Pandolfi C, Ottria L, Mampieri G, et al. Maxillary cyst: description of a clinical case. Oral Implantol. 2009;2(2):28-33. 2. Valois CRA, Costa-Júnior ED. Periapical cyst repair after nonsurgical endodontic therapy - case report. Braz Dent J. 2005;16(3):254-258.

3. Lofthag-Hansen S, Huumonen S, Grondahl K, Grondahl HG. Limited conebeam CT and intraoral radiography for the diagnosis of periapical pathology. Oral Surg Oral Med Oral Pathol Oral Radiol Endod. 2007;103(1):114-119.

4. Rosenberg PA, Frisbie J, Lee J, Lee K, Frommer H, Kottal S, et al. Evaluation of pathologists (histopathology) and radiologists (cone beam computed tomography) differentiating radicular cysts from granulomas. J Endod. 2010;16(3):423-8.

5. Peters E, Lau M. Histopathologic examination to confirm diagnosis of periapical lesions: a review. J Can Dent Assoc. 2003;69(9):598-600.

6. Kadam NS, Ataide Ide N, Raghava P, Fernandes M, Hede R. Management of large radicular cyst by conservative surgical approach: a case report. J Clin Diagn Res. 2014;8(2):239-41.

7. Brondum N, Jensen VJ. Recurrence of keratocysts and decompression treatment. A long-term follow-up of forty-four cases. Oral Surg Oral Med Oral Pathol. 1991;72(3):265-9.

8. Santos Soares SM, Brito-Júnior M, de Souza FK, Zastrow EV, Cunha CO, Silveira FF, et al. Management of Cyst-like Periapical Lesions by Orthograde Decompression and Long-term Calcium Hyd roxide/Chlorhexidine Intracanal Dressing: A Case Series. J Endod. 2016;42(7):1135-41.

9. Maalouf EM, Gutmann JL. Biological perspectives on the non-surgical endodontic management of periradicular pathosis. Int Endod J. 1994; 27(3): 154-62.

10. Bhaskar SN. Nonsurgical resolution of radicular cysts. Oral Surg Oral Med Oral Pathol. 1972;34(3):458-68.
11. Bodner L. Cystic lesions of the jaws in children. Int J Pediatr Otorhinolaryngol. 2002;62(1):25-9.

12. Moiseiwitsch JR, Trope M. Nonsurgical root canal therapy treatment with apparent indications for root-end surgery. Oral Surg Oral Med Oral Pathol Oral Radiol Endod. 1998;86(3):335-40.

13. Lizio G, Sterrantino AF, Ragazzini S, Marchetti C. Volume reduction of cystic lesions after surgical decompression: a computerised three-dimensional computed tomographic evaluation. Clin Oral Investig. 2013;17(7):1701-8.

14. Uloopi KS, Shivaji RU, Vinay C, Shrutha SP, Chandrasekhar R. Conservative management of large radicular cysts associated with non-vital primary teeth: a case series and literature review. J Indian Soc Pedod Prev Dent. 2015;33(1):53-6.

15. Kolokythas A, Schlieve T, Miloro M. Simple method for securing a decompression tube for odontogenic cysts and tumors: a technical note. J Oral Maxillofac Surg. 2011;69(9):2392-5.

16. Swantek JJ, Reyes MI, Grannum RI, Ogle OE. A technique for long term decompression of large mandibular cysts. J Oral Maxillofac Surg. 2012;70(4):856-9.

17. Costa FW, Carvalho FS, Chaves FN, Soares EC. A suitable device for cystic lesions close to the tooth-bearing areas of the jaws. J Oral Maxillofac Surg. 2014;72(1):96-8.

18. Oliveros-Lopez L, Fernandez-Olavarria A, Torres-Lagares D, SerreraFigallo MA, Castillo-Oyagüe R, Segura-Egea JJ, et al. Reduction rate by decompression as a treatment of odontogenic cysts. Med Oral Patol Oral Cir Bucal. 2017;22(5):643-50.

19. Lizio G, Corinaldesi G, Bianchi A, Marchetti C. Successful resolution of juvenile paradental cysts after marsupialization in five consecutive patients. Aust Dent J. 2011;56(4):427-32. 
20. Vidhale G, Jain D, Jain S, Godhane AV, Pawar GR. Management of Radicular Cyst Using Platelet-Rich Fibrin \& Iliac Bone Graft - A Case Report. J Clin Diagn Res. 2015;9(6):34-6.
21. Enislidis G, Fock N, Sulzbacher I, Ewers R. Conservative treatment of large cystic lesions of the mandible: a prospective study of the effect of decompression. Br J Oral Maxillofac Surg. 2004;42(6):546-50.

\section{Mini Curriculum and Author's Contribution}

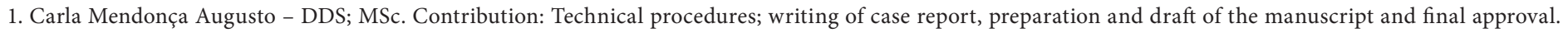
ORCID: 0000-0002-3323-1601

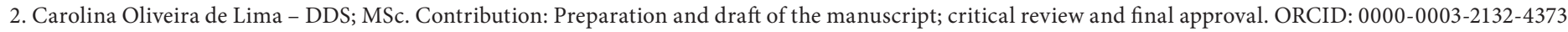

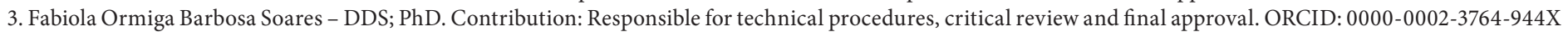

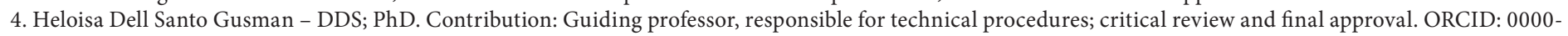
0002-7470-6650

Submitted: 09/22/2020 / Accepted for publication: 09/24/2020

Corresponding author

Carolina Oliveira de Lima

E-mail: c.oliveiradelima@yahoo.com.br 